\author{
R. Jahn', M. Dressel ${ }^{2}$, W. Neu², K. H. Jungbluth
}

Abteilung Unfallchirurgie ${ }^{i}$ (Direktor: Prof. Dr. K. H. Jungbluth), Universitätskrankenhaus Hamburg-Eppendorf und Laser-Laboratorium Göttingen e.V.2 (Direktor: Priv.-Doz. Dr. Gerhardt)

\title{
Abtragung von biologischem Hartgewebe mit dem Excimerlaser
}

Die durchgeführten Experimente zeigen die Schneid- und Bohrmöglichkeiten einer Excimerlaser-Faser-Kombination am Knochen- und Meniskusgewebe auf. Für die Abtragungsvorgänge am Meniskus konnten bereits akzeptable Operationsgeschwindigkeiten erzielt werden. An den Hart- wie auch an den Weichgeweben hinterlassen die Ablationsvorgänge dabei nur begrenzte, mikroskopisch eben erkennbare Zell- und Gewebsschädigungen. Eine weitere Steigerung der Abtragrate für Hartgewebe erscheint technisch durch eine Erhöhung der Transmissionsenergie via Faser erreichbar zu sein. Die damit verbundene Beschleunigung der Gewebeablation und der Schneidevorgänge rückt Laserstrahlung mit hoher Energiedichte wieder in das Interesse der Unfallchirurgen.

\begin{abstract}
Ablation of hard biological tissue with the excimer laser
Cutting and drilling of bone- and meniscus tissue were performed using a XeCl-excimer laser combined with a tapered fiber. Ablation speed on meniscus tissue is already sufficient, the thermal damage of the adjacent tissue is minimal. Increasing of energy transmission through special fibers promises higher ablation rates also on hard biological tissue and that promoted the interest in lasers again for accident surgeons.
\end{abstract}

\section{Einleitung}

Seit den 60er Jahren hat der Laser einen schnellen Einzug in die Medizin gehalten, trotzdem blieb die Bearbeitung des biologischen Hartgewebes stets eine unüberwindliche Hürde. Für das Schneiden von Knochen wurden hohe Energien gebraucht, die sich bei Anwendung der herkömmlichen Laser $\left(\mathrm{CO}_{2}\right.$, Nd:YAG) aus dem IR-Bereich in große thermische Nebenwirkungen umsetzen und zu ausgedehnten Nekrosezonen führten, die bereits makroskopisch an der Karbonisierung des Gewebes erkennbar waren.

Erwartungsgemäß waren die Heilungsraten gestört, und es schien, als stelle der Laser für die Fachgebiete Unfallchirurgie und Orthopädie im Gegensatz zu

Eingang des Manuskripts: 21. 3. 1991

Annahme des Manuskripts: 22. 9. 1991 anderen wie zum Beispiel Ophthalmologie oder Neurochirurgie keine Alternative zu den konventionellen Schnittechniken dar.

Die Entwicklung des Excimerlasers zeigt mit dem athermischen Abtragungsvorgang (Photoablation) neue Wege auf. Im Rahmen industrieller Fertigungsverfahren ist es möglich geworden, in härtesten Materialien (Keramik, Stahl, Glas, Diamant) auf präzise Weise Oberflächenmarkierungen, Schnitte und Bohrungen ohne wesentliche thermische Nebenerscheinungen vorzunehmen. Der hierbei zur flexiblen Führung des Strahls meist verwendete Spiegelarm ist jedoch für viele medizinische Einsatzbereiche nicht geeignet, erwünscht wäre statt dessen die Leitung durch Glasfasern. Für die meisten Anwendungen eines Lasers auf unfallchirurgischem Gebiet ist eine solche Strahlführung durch eine flexible Faser sogar unabdingbare Voraussetzung, da Verbesserungen der bisherigen Operationsmethodik vor allem in der Weiter- 


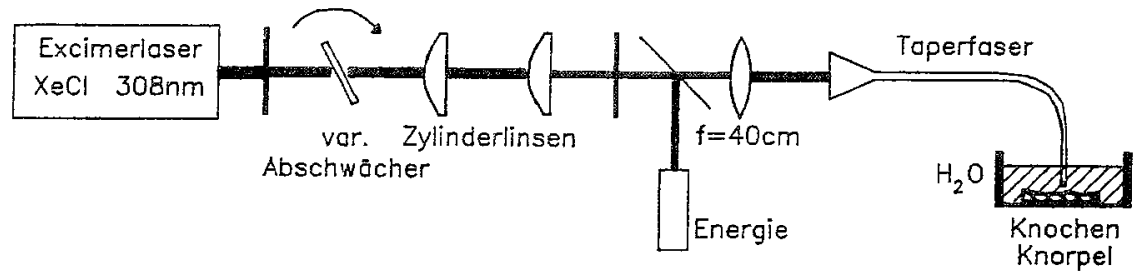

Abbildung 1. Experimenteller Aufbau

entwicklung zu einer ,,minimal-invasiven Chirurgie "zu sehen sind. Das bedeutet die zunehmend stärkere Einbindung endoskopischer Verfahren in die Operationstechnik.

Schnittversuche mit dem Excimerlaser am Knochen scheiterten bisher an der begrenzten Transmissionsfähigkeit von Lichtleitfasern für UV-Strahlung. Außerdem wurde die geringe Eindringtiefe der Strahlung als Nachteil angesehen. Die erhaltenen Leistungsdichten am Ablationsort waren zu gering und die Abtraggeschwindigkeit mit bisher höchsten $50 \mu \mathrm{m} / \mathrm{s}$ am Knochengewebe für einen operativen Einsatz zu niedrig.

Mit einem speziellen Einkoppelverfahren (Abbildung 1) ist es uns gelungen, über die bisher begrenzende Oberflächenzerstörung der Faser hinaus hochenergetische UV-Laserpulse zu übertragen. Durch die Verwendung von Taperfasern, das heißt Quarzfasern

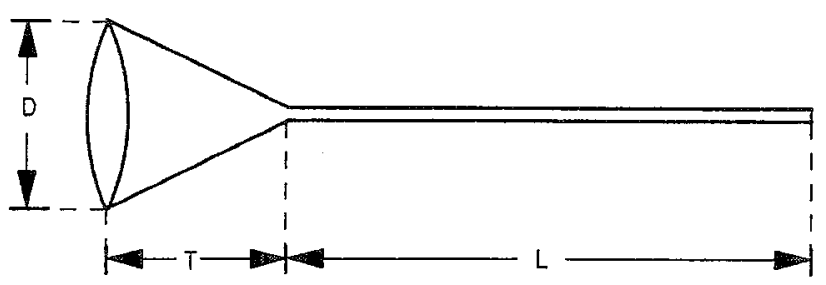

Abbildung 2. Taperfaser. $\mathrm{D}=5 \mathrm{~mm}, \mathrm{~T}=60 \mathrm{~mm}, \mathrm{~L}=1700 \mathrm{~mm}$.

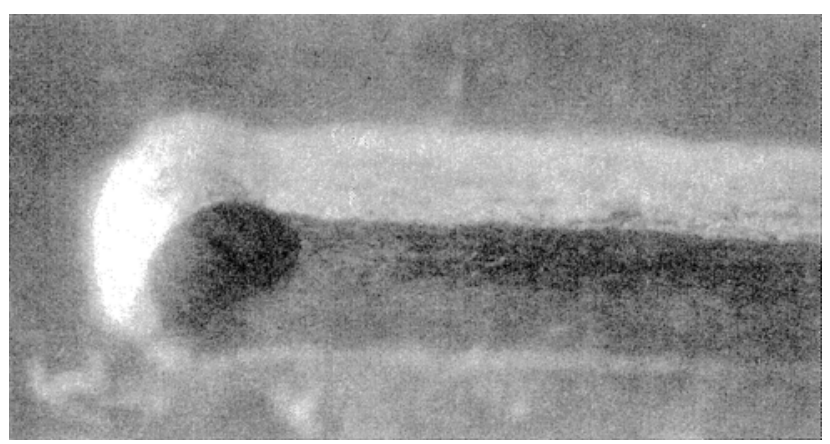

Abbildung 3. Knochenschnitt. Glatte, gratlose Schnittränder ohne Karbonisation. mit einem konischen Einkoppelstück des zirka zehnfachen Durchmessers [1], ist es möglich, $250 \mathrm{~mJ}$ durch eine Faser mit $1000 \mu \mathrm{m}$ Kerndurchmesser zu transportieren; das heißt Energiedichten von über $30 \mathrm{~J} / \mathrm{cm}^{2}$. Die Strahleinkopplung ist bei Taperfasern (Abbildung 2) ausgesprochen unkritisch. Für die medizinische Anwendung steht hiermit wesentlich mehr Energie am Faserende zur Verfügung, und es wird dadurch eine präzise und deutliche Abtragung auch am Knochengewebe erreicht (Abbildung 3).

\section{Zielsetzung}

Die Zielsetzung unseres Versuches bestand darin, Bedingungen zur Optimierung von Meniskus- und Knochenablationsraten zu schaffen, indem die Wechselwirkungen zwischen Laserparametern und Gewebe im einzelnen untersucht wurden. Hierbei wurde der Einfluß der Energie, der Repetitionsrate, der Energiedichte, der Pulsdauer, der Leistungsdichte, des Faserdurchmessers, des Abstands und des umgebenden Mediums untersucht. Es interessierten die Tiefe der erzielten Schnitte und Bohrungen, die Ablationsrate, die Schnitt- bzw. Bohrgeschwindigkeit, die Qualität der Schnittoberfläche, mögliche Karbonisation, die Temperaturentwicklung und -verteilung. Weiterhin sollten Feststellungen zur Behandlung des fasergeführten Excimerlasers getroffen werden.

\section{Material und Methode}

Excimerlaser (XeCl) der Wellenlänge $308 \mathrm{~nm}$ (Fa. Lambda Physik, Göttingen): EMG 1003 i (Pulsdauer 28 ns), LPX 605 iCC (Pulsdauer 60 ns) und EMG 602 (Pulsdauer 250 bis $300 \mathrm{~ns}$ ),

Taperfasern (Fa. Heraeus Quarzglas): Länge $200 \mathrm{~cm}$, Kerndurchmesser $400 \mu \mathrm{m}, 600 \mu \mathrm{m}, 1000 \mu \mathrm{m}$,

Rippenknochen: Meniskus von frischen Schlachttieren (Schwein, Rind).

(Versuchsaufbau: siehe Abbildungen 4 und 5.)

Die einzelnen Parameter variierten wie folgt: 


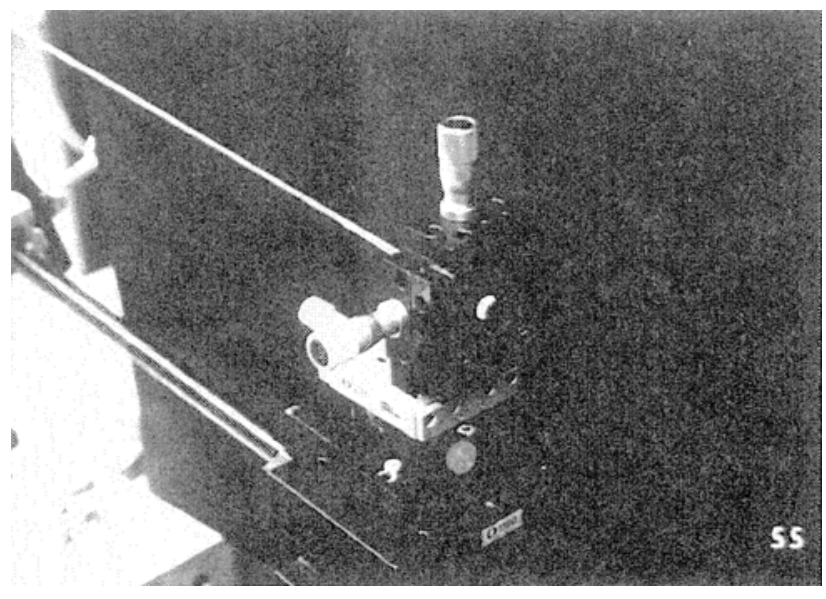

Abbildung 4. Aufbau zur Einkopplung des Excimerlaserpulses in die Taperfaser.

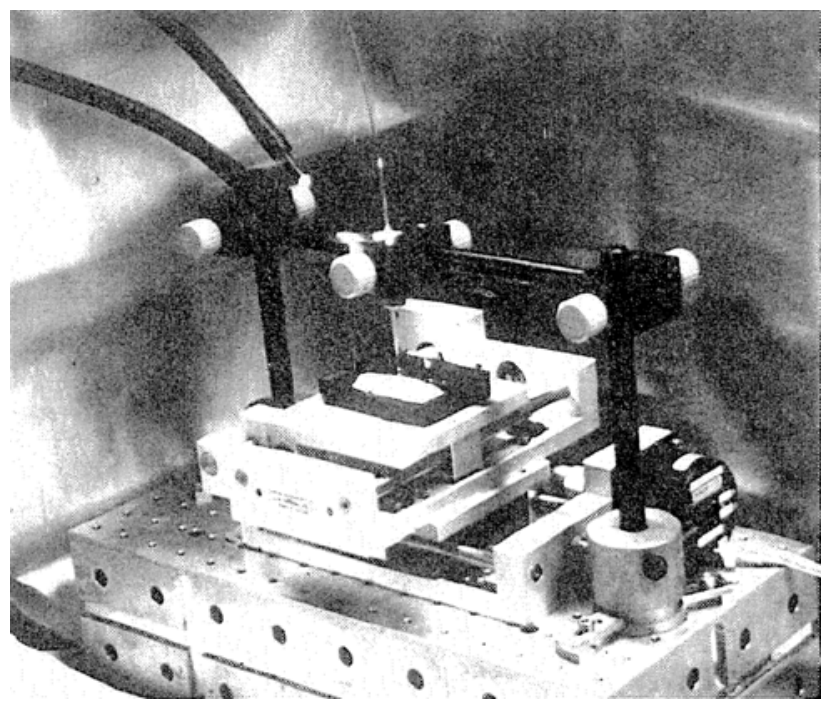

Abbildung 5. Fixiertes Faserende über dem Präparat, welches durch einen computergesteuerten Schrittmotor unter der Faser bewegt wird.

Ausgangsenergie von $20 \mathrm{~mJ}$ bis $70 \mathrm{~mJ}$; Repetitionsrate von 10 bis $100 \mathrm{~Hz}$; Pulsbreite: $28 \mathrm{~ns}, 60$ ns, $300 \mathrm{~ns}$; Energiedichte von $2,1 \mathrm{~J} / \mathrm{cm}^{2}$ bis $20 \mathrm{~J} / \mathrm{cm}^{2}$; Faserdurchmesser $400 \mu \mathrm{m}, 600 \mu \mathrm{m}, 1000 \mu \mathrm{m}$; Betrahlungszeit zum Beispiel 60-90-120s. Die Ablationen wurden als Bohrungen und Schnitte vorgenommen.

\section{Ergebnisse}

\section{Ablation am Knochen}

Bei niedriger Energiedichte sind die Ablationsarten bei Kurzpulslaser ( $28 \mathrm{~ns}$ ) und Langpulslaser ( $33 \mathrm{~ns}$ ) annähernd gleich (Abbildung 6 und 7). In höheren Bereichen (Energiedichte $6 \mathrm{~J} / \mathrm{cm}^{2}$ ) nimmt die Abtragrate bei Verwendung des Langpulslasers deutlich zu. Es wer-

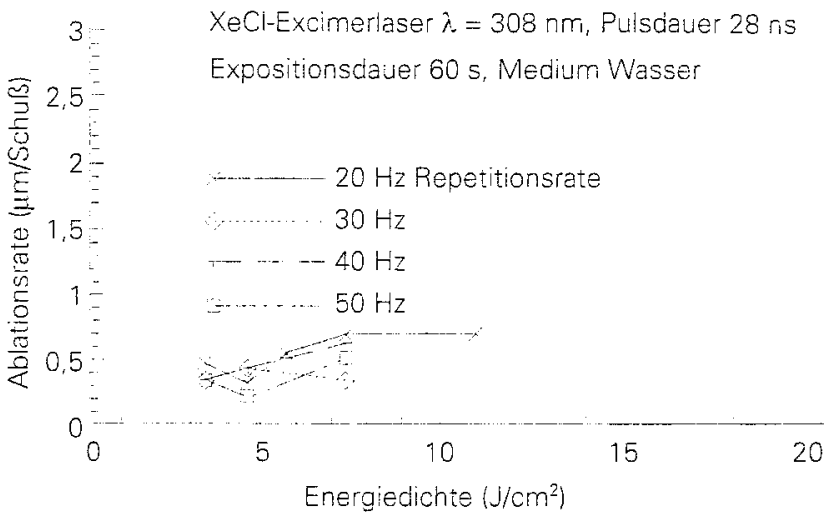

Abbildung 6. Abhängigkeit der Lochtiefe von der Repetitionsrate und der Energie bei Bohrungen unter $60 \mathrm{~s}$ Strahlenexposition eines 28-ns-Excimerlasers (Wellenlänge $308 \mathrm{~nm}$ ), gefüht durch eine $1000-\mu \mathrm{m}$-Faser.

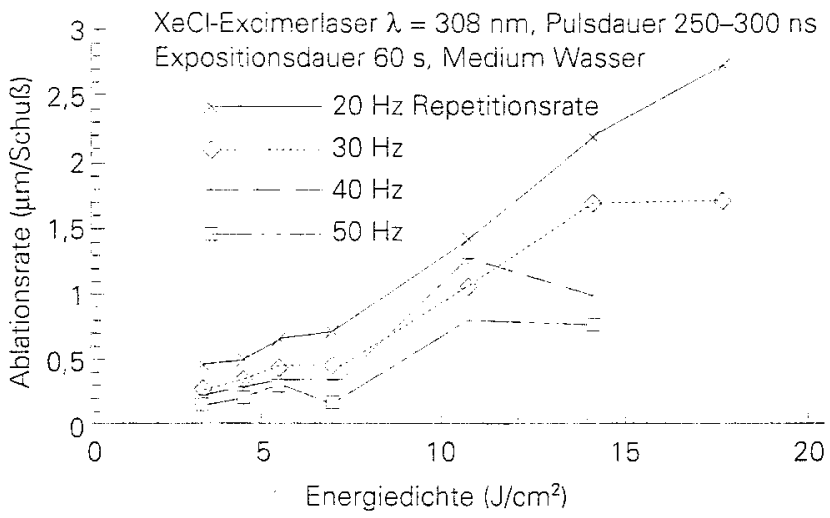

Abbildung 7. Abhängigkeit der Lochtiefe von der Repetitionsrate und der Energie bei Bohrungen unter 60 s Strahlenexposition eines 300-ns-Excimerlasers (Wellenlänge $308 \mathrm{~nm}$ ), geführt durch eine 1000-um-Faser.

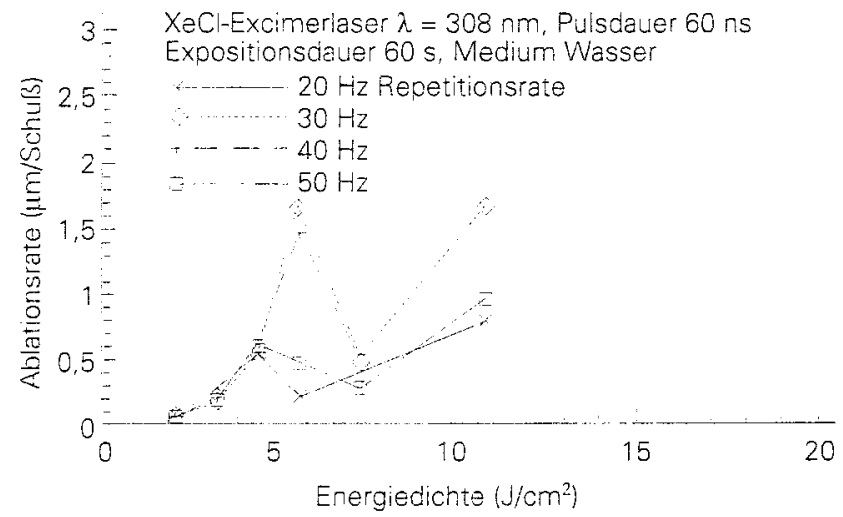

Abbildung 8. Abhängigkeit der Lochtiefe von der Repetitionsrate und der Energie bei Bohrungen unter $60 \mathrm{~s}$ Strahlenexposition eines 60-ns-Excimerlasers (Wellenlänge $308 \mathrm{~nm}$ ), geführt durch eine 1000-um-Faser. 
den Repetitionsraten bis $50 \mathrm{~Hz}$ auch noch bei einer Energiedichte über $8 \mathrm{~J} / \mathrm{cm}^{2}$ toleriert. Hohe Ablationswerte weist der Mittelpulslaser ( 60 ns) bei Energiedichtenum $5,4 \mathrm{~J} / \mathrm{cm}^{2}$ auf. Von der Faser werden bei dieser Pulsbreite die mittleren Repetitionsraten (30 und $40 \mathrm{~Hz}$ ) sehr gut toleriert (Abbildung 8).

Die erreichten Energiedichten liegen bei zirka $20 \mathrm{~J} / \mathrm{cm}^{2}$ (Pulslänge 250 bis $300 \mathrm{~ns}$ ) und $15 \mathrm{~J} / \mathrm{cm}^{2}$ (Pulslänge $60 \mathrm{~ns}$ ). Knochenbohrungen verlaufen bei einer maximalen Geschwindigkeit von $0,08 \mathrm{~mm} / \mathrm{s}$, das entspricht einer Ablationsrate von $>2 \mu \mathrm{m} /$ Puls. Knochenschnitte mit Tiefen bis zu 4,5mm zeigen glatte Schnittkanten und keine Karbonisation. Ein Knochendurchschnitt von $754,5 \mathrm{~mm}$ Fäche, dargestellt in Abbildung 9, ist makroskopische in Farbe und Oberflächenbeschaffenheit kaum verändert.

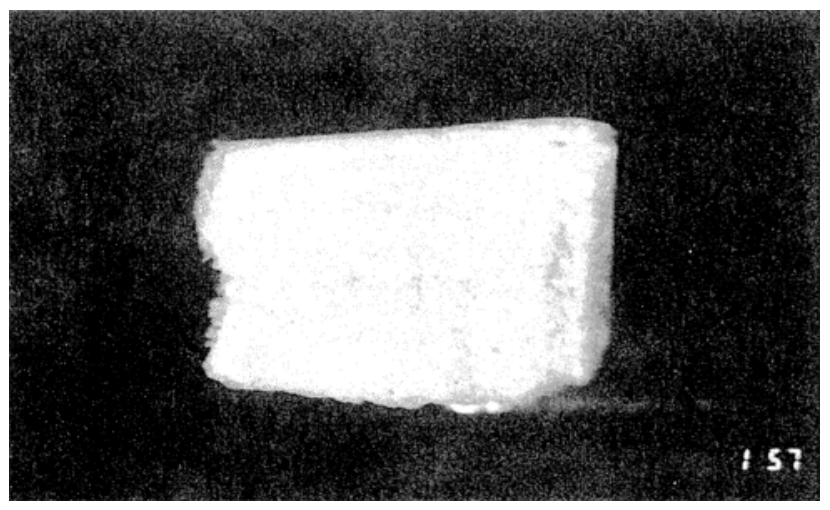

Abbildung 9. Laserdurchschnitt eines Knochens. Keine Karbonisation.

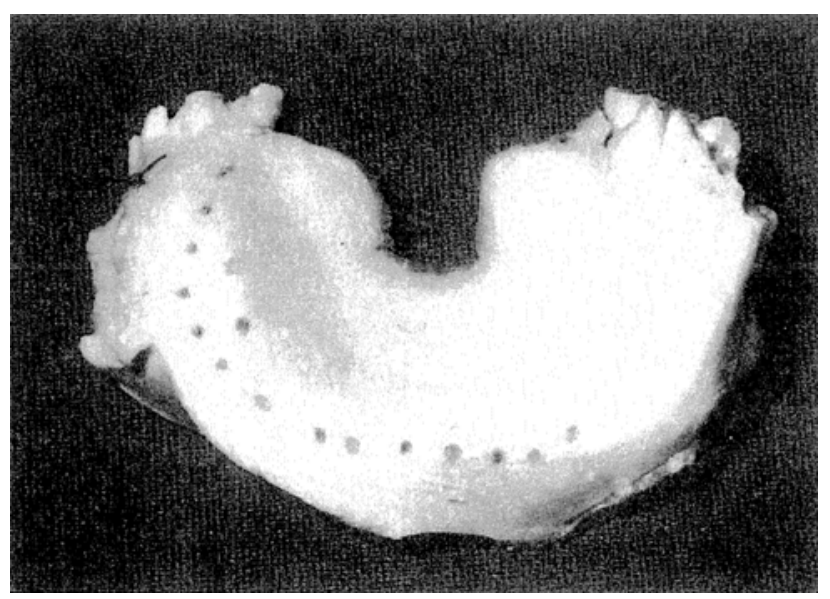

Abbildung 10. Meniskusbohrungen (bis zu einer Parametereinstellung von $50 \mathrm{~mJ}, 4 \mathrm{~Hz}$ ).

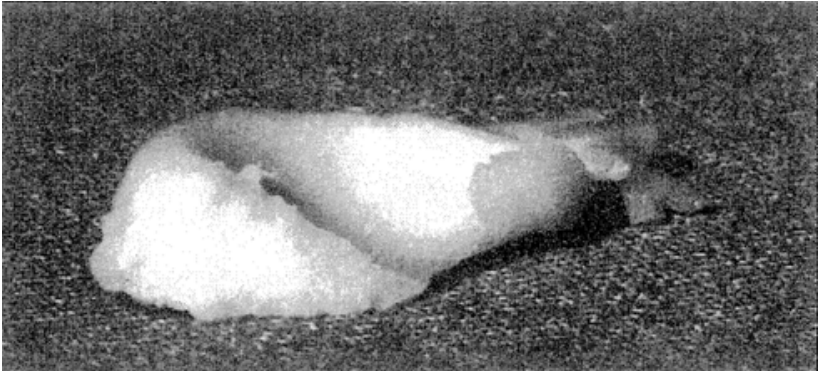

Abbildung 11. Experimentelle Meniskusteilresektion.

\section{Ablation am Meniskusgewebe}

Bei Bohrungen im Rindermeniskus ist eine Bohrgeschwindigkeit von 2 bis $6 \mathrm{~mm} / \mathrm{s}$ zu verzeichnen (Abbildung 10).

Die Durchtrennung eines Meniskus (Fläche 4513mm) wurde in 111s erreicht. Das Gewebe ist an der Schnittfläche etwas aufgelockert. Keine Karbonisierungen. Kein Gewebeschrumpfen während der Laserapplikation (Abbildung 11).

\section{Diskussion}

Bei den fasergeführten Laserexpositionen (Excimerlaser $308 \mathrm{~nm}$ Wellenlänge, Pulsbreite $28 \mathrm{~ns}$ und $300 \mathrm{~ns}$ ) stellt sich zunächst eine deutliche Überlegenheit des Langpulslasers (Spezialaufbau eines Experimentierlasers) gegenüber dem Standardpuls ein. Dies zeigt sich in einer wesentlichen Verbesserung der Haltbarkeit der Faser und kommt in den Abbildungen 6 und 7 in der Gegenüberstellung der erreichten Lochtiefen bei Anhebung der Repetitionsraten sichtbar zum Ausdruck. Die Begrenzung in diesen Experimenten war regelmäßig die Zerstörung des Faserendes, nicht das Erreichen des Sättigungsbereiches der Ablation. Da diese Versuche im Hinblick auf einen klinischen Einsatz des Excimerlasers durchgeführt werden, muß auch der momentane Nachteil des Langpulslasers für die Klinik gesehen werden. Er ist nicht als Seriengerät erhältlich.

Es zeigte sich dafür der LPX 605 iCC mit einer Pulslänge von 60 ns als ein guter Kompromiß zwischen den physikalischen Eigenschaften bezuiglich der optischen Zestörung der Fasern einerseits und Bedienungskomfort und Spezifikation andererseits. Aufgrund der besseren Ablationseigenschaften kürzerer Pulse wurden mit dem 60-ns-Excimerlaser in den Bereichen von 30 bis $40 \mathrm{~mJ}$ Eingangsenergie sogar größere Lochtiefen 
erreicht als bei Einsatz des Langpulslasers von $300 \mathrm{~ns}$ Pulsbreite bei gleicher Energiedichte (Abbildung 8).

Repetitionsraten über $50 \mathrm{~Hz}$ werden bei einer Pulsbreite von 60 ns von der Faser bei Knochenablationen zur Zeit noch nicht toleriert. Hier könnte ein größerer Abstand zwischen Faser und Gewebe Verbesserungen bringen.

Erste Untersuchungen uber das Temperaturausbreitungsverhalten bei Hartgewebsablation mit dem Excimerlaserstrahl deuten auf Möglichkeiten hin, durch entsprechende Variation von Energie und Repetitionsrate auch Temperaturen bis zu $100^{\circ} \mathrm{C}$ direkt am Faserende im Gewebe zu erzeugen. Eine Koagulation von kleinen Blutgefäßen wäre somit nicht mehr ausgeschlossen.

\section{Danksagung}

Die Autoren danken den Firmen Lambda Physik Göntingen und Heraeus Quarzglas Hanau für die großzïgige Unterstïtzung der Experimente.

\section{Literatur}

1. Greulich, K. O., H. Hitzler, N. Leclerc, J. Wolfrum, K. F. Klein: Transport von leistungsstarken UV-Laserpulsen durch Lichtleiter mit variablem Querschnitt (Taper). Laser u. Optoelektronik 20 (1988), 58

2. Jahn, R, M. Dressel, H. Fabian, H. Gerhardt, J. Kesper, K. F. Klein, H. U. Langendorff, W. Neu, U. Sowada, K. H. Jungbluth: Excimerlaser und Taperlaser - ein effizientes Instrument für die Ablation von Knochen und Knorpelgewebe. Laser Med. Surg. 6 (1990), 77.

3. Jahn, R., M. Dressel, H. Fabian, K. F. Klein, H. U. Langendorff, W. Neu, K. H. Jungbluth: Bohren an Knochen mit fasergeführtem Excimerlaserstrahl. Laser Med. Surg. 6 (1990), 172.

Für die Verfasser: Priv.-Doz. Dr. Renate Jahn, Abteilung für Unfall-und Wiederherstellungschirurgie, Chirurgische Klinik und Poliklinik, Universitatskrankenhaus Eppendorf, Martinistraße 52, D-2000 Hamburg 20. 